\title{
Effect of pre-coating with methyl methacrylate containing UV photoinitiators on the bond strength of poly(ether ether ketone)
}

\author{
Seigo OKAWA'1 , Yujin AOYAGI², Tatsuya KIMURA² and Kenji IZUMI ${ }^{1}$ \\ ${ }^{1}$ Division of Biomimetics, Faculty of Dentistry \& Graduate School of Medical and Dental sciences, Niigata University, 2-5274, Gakkocho-dori, Chuo- \\ ku, Niigata 951-8514, Japan \\ ${ }^{2}$ Division of Bio-Prosthodontics, Faculty of Dentistry \& Graduate School of Medical and Dental sciences, Niigata University, 2-5274, Gakkocho-dori, \\ Chuo-ku, Niigata 951-8514, Japan \\ Corresponding author, Seigo OKAWA; E-mail: sokawa@dent.niigata-u.ac.jp
}

\begin{abstract}
This study investigates the effect of pre-coating with methyl methacrylate (MMA) containing ultraviolet (UV) photoinitiators on the bond strength of poly(ether ether ketone) (PEEK). Cylindrical PEEK blocks were irradiated with $365 \mathrm{~nm}$ UV light for 5-20 s after they were coated with MMA containing 0.4-3.0 wt\% UV photoinitiators: [1-phenyl-1,2-propanedione (PPD)], [diphenyl(2,4,6trimethylbenzoyl) (TMDPO)], and [phenyl bis(2,4,6-trimethylbenzoyl) phosphine oxide (BTMPO)]. Pre-coated PEEKs were bonded to PEEK blocks with a MMA-based adhesive resin. The shear bond strength was measured using a universal testing machine. Secondary electron images were captured to observe failure surfaces. The data were analyzed with one- and two-way ANOVA and Tukey's post hoc tests $(p<0.05)$. The highest bond strength $(20.7 \pm 5.1 \mathrm{MPa})$ was observed for pre-coating with MMA containing 0.4 wt\% BTMPO, for $20 \mathrm{~s}$ of UV irradiation. Cohesive failure of the adhesive resin was observed. The use of this pre-coating led to improved bonding performance of PEEK.
\end{abstract}

Keywords: Poly(ether ether ketone), UV photoinitiator, Shear bond strength, Surface modification

\section{INTRODUCTION}

Poly(ether ether ketone) (PEEK) has applications in the dental and medical fields as a type of material that recovers the loss of body functions, owing to its excellent biocompatibility ${ }^{1-3)}$. Recently, several dental prostheses, implants, and medical devices composed of PEEKs have been fabricated by computer-aid design/ computer-aided manufacturing $(\mathrm{CAD} / \mathrm{CAM})$ and 3-D printing techniques ${ }^{4,5)}$. PEEKs are polymeric materials with excellent mechanical properties, and their elastic modulus is similar to that of bone. Therefore, PEEKs are capable of minimizing the bone resorption associated with the stress shielding property of a rigid system ${ }^{2)}$.

PEEK is chemically inert, and hence, possesses poor adhesive properties. To facilitate their widespread use as biomaterials, the adhesive properties of PEEK and its fiber composites need to be improved.

In previous studies ${ }^{6-21)}$, the bond strengths of PEEK and fiber-reinforced PEEKs were investigated after the modification of their surfaces by sandblasting ${ }^{6-11)}$, chemical treatment ${ }^{6,7,9,10,12,13)}$, and plasma ${ }^{14-18)}$ and UV ${ }^{19-21)}$ irradiation. These studies showed that the bond strengths of PEEK and fiber-reinforced PEEKs increase following their surface modification, and are slightly higher than $10 \mathrm{MPa}$ in dental applications. However, surface modifications in chairside dentistry are limited.

In this study, we particularly focus on the dental curing light system. Ultraviolet (UV) light is used to modify the PEEK surface instead of visible light ${ }^{19-21)}$ because the wavelength of UV light is shorter than that of visible light, and thus, the light energy from UV light is higher than that from visible light. Therefore, the
PEEK surface was modified by the UV light energy.

The use of visible light-activated polymers, however, has been extended to dental materials and several presentations are available. All contain diacrylate resin monomers, suitable pigments and visible light initiators which produce the free radicals needed to promote addition polymerization ${ }^{22}$. We note that a methyl methacrylate (MMA) is used as a solvent for UV photoinitiators because a MMA monomer is a clear and colorless liquid. After the PEEK surface was pre-coated with a MMA monomer containing a UV photoinitiator, the surface was evenly irradiated with UV light at a wavelength of $365 \mathrm{~nm}$. Free radicals can be subsequently produced and causes the polymerization of MMA to form a polymethylmethacrylate (PMMA) film. It is hypothesized that PMMA formed by the polymerization of MMA is strongly bonded to the modified PEEK surface.

The objective of this study is to evaluate the effect of UV initiators, their concentrations, and UV irradiation time on the bond strength of PEEK.

\section{MATERIALS AND METHODS}

\section{Specimens}

PEEK rods (Ketron 1000 PEEK, Quadrant Polypenco Japan, Tokyo, Japan) with a diameter of $10 \mathrm{~mm}$ were cut into cylindrical PEEK blocks with a height of approximately $5 \mathrm{~mm}$ using a cutting unit. Half of the blocks were embedded in a thermal curing resin to prepare adherend blocks. The ends of the flat surface of the adherends and cylindrical PEEK blocks were polished with 1000-grit $\mathrm{SiC}$ waterproof paper under 
running tap water. Both PEEK blocks were cleaned ultrasonically in distilled water for $10 \mathrm{~min}$, and were air-dried completely.

\section{Pretreatment}

Considering photoinitiator stability and the wide use of photoinitiators in free radical polymerization for dentistry ${ }^{23)}$, we focused on the following three photoinitiators; [1-phenyl-1,2-propanedione (PPD) (Tokyo Chemical Industry, Tokyo, Japan)], [diphenyl (2,4,6-trimethylbenzoyl) phosphine oxide (TMDPO) (Tokyo Chemical Industry)], and [phenyl bis(2,4,6trimethylbenzoyl) phosphine oxide (BTMPO) (Tokyo Chemical Industry)]. These photoinitiators were dissolved in MMA monomer (FUJIFILM WAKO Pure Chemical, Osaka, Japan) to prepare pre-coating solutions of different concentrations ranging from 0.4 to $3.0 \mathrm{wt} \%$.

The prepared solution was pre-coated on both, the adherend and the cylindrical PEEK block surfaces, with a brush. Subsequently, the surfaces were individually exposed to UV light (EXECURE 4000, HOYA, Japan) of wavelength $365 \mathrm{~nm}$ for $20 \mathrm{~s}$.

\section{Absorption peaks for the prepared MMA solutions}

A UV-visible programmable spectrophotometer (DU640, Beckman Instruments, CA, USA) with an operational range of 200 to $700 \mathrm{~nm}$ was employed to measure the maximum wavelength of the prepared $0.4 \mathrm{wt} \%$ solutions.

\section{Fourier transform infrared (FTIR) spectroscopy}

The pretreated specimen with $0.4 \mathrm{wt} \%$ BTMPO was analyzed using a Fourier transform infrared (FTIR) spectrometer (FTIR-7300, JASCO, Hachioji, Japan) to verify the formation of PMMA. A total of 256 scans were collected at a resolution of $4 \mathrm{~cm}^{-1}$, and the spectrum was obtained in the reflectance mode in the region of 4400 to $400 \mathrm{~cm}^{-1}$.

\section{Bonding procedure}

A thin polyethylene terephthalate seal with a circular hole of diameter $6 \mathrm{~mm}$ was placed on the pretreated adherend specimen surface to maintain the adhesive area constant. The specimen was bonded to the cylindrical PEEK block using a MMA adhesive resin (Super-Bond C\&B, Sun Medical, Shiga, Japan) according to the manufacturer's instructions $(n=5)$. After bonding, the specimens were left for $1 \mathrm{~h}$ under atmospheric conditions and then stored in distilled water at $37 \pm 1^{\circ} \mathrm{C}$ for $24 \mathrm{~h}$.

\section{Bond strength test}

The bonded specimens were stressed in shear compression using a universal testing machine (AE1000, Shimadzu, Kyoto, Japan) at a crosshead speed of $1.0 \mathrm{~mm} / \mathrm{min}$ until failure. A total of five specimens were examined under each condition.

\section{Observation of failure surfaces}

The secondary electron (SE) imaging mode of an electron beam micro-analyzer (EPMA8710, Shimadzu) was utilized to observe the failure surface. The failure surfaces were coated with $\mathrm{AgPd}$ in a sputter coater before observing them.

\section{Statistical analysis}

Data were analyzed with one- and two-way ANOVA (UV photoinitiator versus its concentration and bond strength), and Tukey's post hoc test at a pre-set significance level of 0.05 .

\section{RESULTS}

\section{Spectrum distribution for UV-visible light}

Figure 1 displays the UV-visible light absorption spectra of the 0.4 wt $\%$ pre-coating solutions. The absorption spectra of the solutions revealed that the maximum absorption occurred in the wavelength region of 300 to $500 \mathrm{~nm}$, with a $\lambda_{\max }$ of $415 \mathrm{~nm}$.

\section{FTIR}

The FTIR spectrum of the pretreated specimen is shown in Fig. 2. The peaks are assigned as described elsewhere ${ }^{24)}$. The peak at approximately $3,000 \mathrm{~cm}^{-1}$ is attributed to $\mathrm{C}-\mathrm{H}$ stretching in $\mathrm{CH}_{3}$, as is the peak at $1,385 \mathrm{~cm}^{-1}$. The intense peak at $1,732 \mathrm{~cm}^{-1}$ is assigned to $\mathrm{C}=\mathrm{O}$ double bond stretching, whereas the broad envelope between 1,244 and $1,151 \mathrm{~cm}^{-1}$ is attributed to C-O-C single-bond stretching. The peaks at 1,520, 1,388, and $754 \mathrm{~cm}^{-1}$ are attributed to $\mathrm{C}=\mathrm{C}$ stretching.

\section{Shear bond strengths}

The compressive shear bond strengths of the specimens with different pre-coatings are shown in Fig. 3.

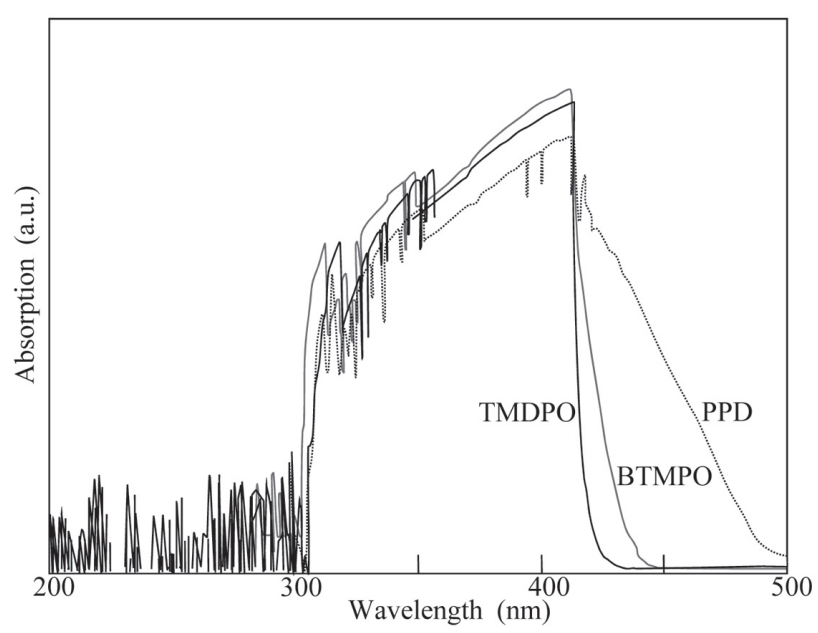

Fig. 1 Spectral distribution of UV-visible light in comparison with the absorption peaks for the prepared $0.4 \mathrm{wt} \%$ solutions.

The absorbance maxima were observed at 415 $\mathrm{nm}$ in the wavelength region of 300 to $500 \mathrm{~nm}$. In this study, the excitation wavelength of the photoinitiators was selected as approximately 365 nm. 


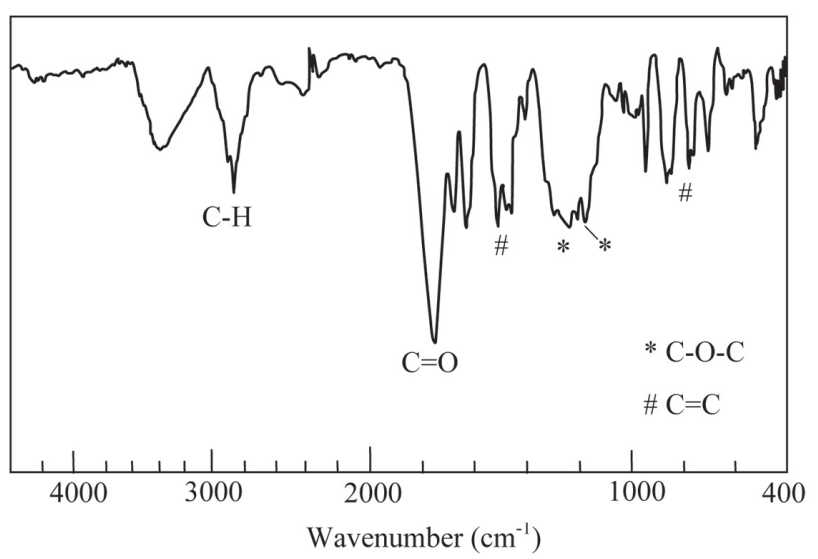

Fig. 2 FTIR spectrum of the specimen pretreated with 0.4 wt\% BTMPO.

The specimen mainly contains carbonyl groups exhibiting a band at $1,732 \mathrm{~cm}^{-1}$, which is attributed to PMMA.

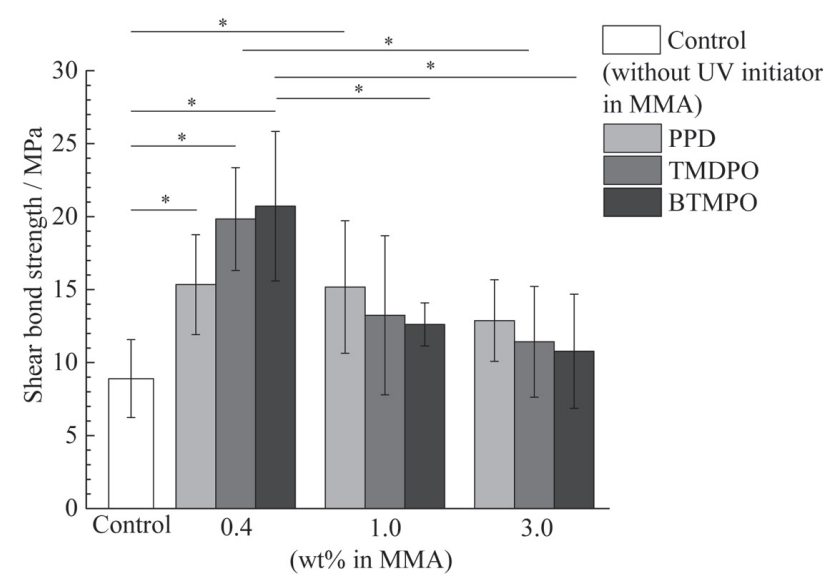

Fig. 3 Compressive shear bond strengths.

Bars indicate standard deviation. Single asterisk indicates $p<0.05$ for specimen comparison. Control: without UV initiators in MMA. UV initiators: PPD, TMDPO, and BTMPO.

Statistically significant differences were found among the specimens pre-coated with $0.4 \mathrm{wt} \%$ PPD, TMDPO, BTMPO, and the control. There was no statistically significant difference in the bond strength associated with changes in the concentration of PPD. However, the bond strength of the specimen pre-coated with 0.4 wt\% TMDPO was significantly higher than that of the specimen pre-coated with $3.0 \mathrm{wt} \%$ TMDPO. Additionally, the bond strength of the specimen pre-coated with 0.4 wt\% BTMPO was significantly higher than the bond strengths of the specimens pre-coated with 1.0 and 3.0 wt\% BTMPO. The specimens pre-coated with the same concentration of UV photoinitiators exhibited similar bond strengths regardless of the type of UV photoinitiators.
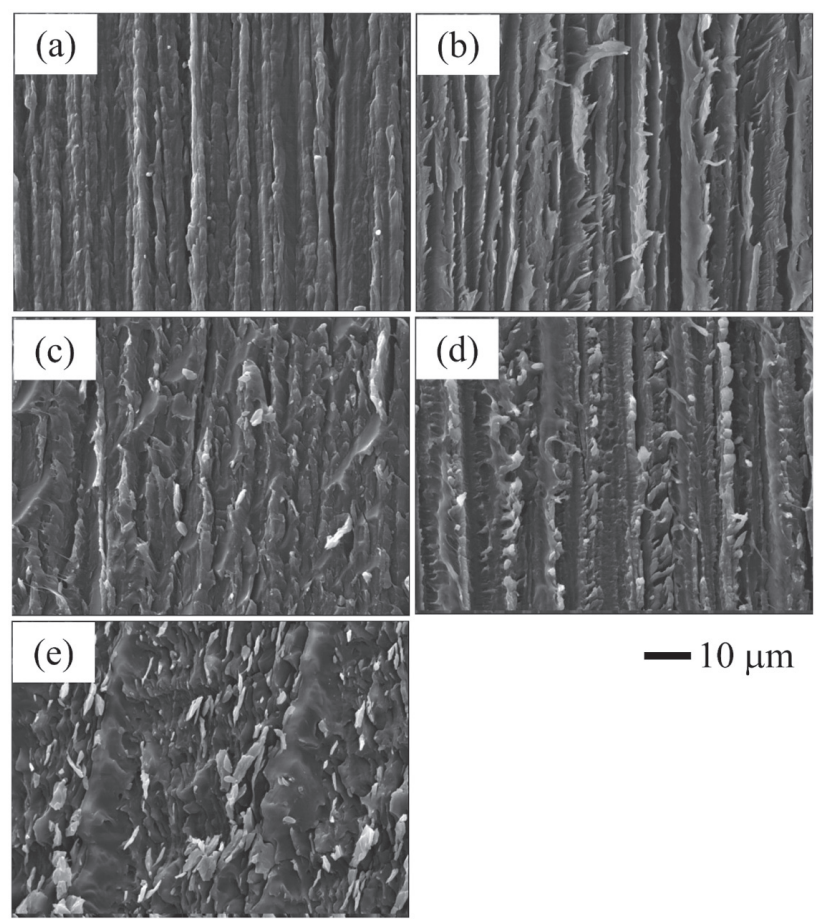

$-10 \mu \mathrm{m}$

Fig. 4 SE images of the fractured PEEK surfaces.

(a) As-polished, (b) Control, (c) 0.4 wt\% PPD, (d) 0.4 wt\% TMDPO, and (e) 0.4 wt\% BTMPO. Polishing streaks were observed in (a), traces of adhesive resin along the polishing streaks were observed in (b), torn resin strips were detected in (c) and (d), no polishing streaks, but cohesive failure, was observed in (e).

\section{Observation of failure surfaces}

Because the bond strengths of the specimens pre-coated with the $0.4 \mathrm{wt} \%$ UV photoinitiator were higher than those of the other specimens, the PEEK failure surfaces under this condition and the polished PEEK surfaces were observed (Fig. 4). In the control, traces of the adhesive resin were detected along the polishing streaks. On the other hand, in the specimens pre-coated with PPD and TMDPO, torn resin strips were observed at the polishing streaks. However, no adhesive resin was present on the flat surfaces of the polishing streaks. In the specimen pre-coated with BTMPO, no polishing streaks were detected, and cohesive failure of the adhesive resin was observed.

\section{Effect of irradiation time on bond strength}

Figure 5 shows the relationship between the UV irradiation time and bond strength for $0.4 \mathrm{wt} \%$ PPD and BTMPO. There was no statistically significant difference between the control and the specimen precoated with PPD when irradiated for $5 \mathrm{~s}$. However, the bond strength of the specimen pre-coated with BTMPO was significantly higher than the bond strengths of the control and the specimen pre-coated with PPD. In contrast, the bond strengths of specimens pre-coated with UV photoinitiators were significantly higher than 


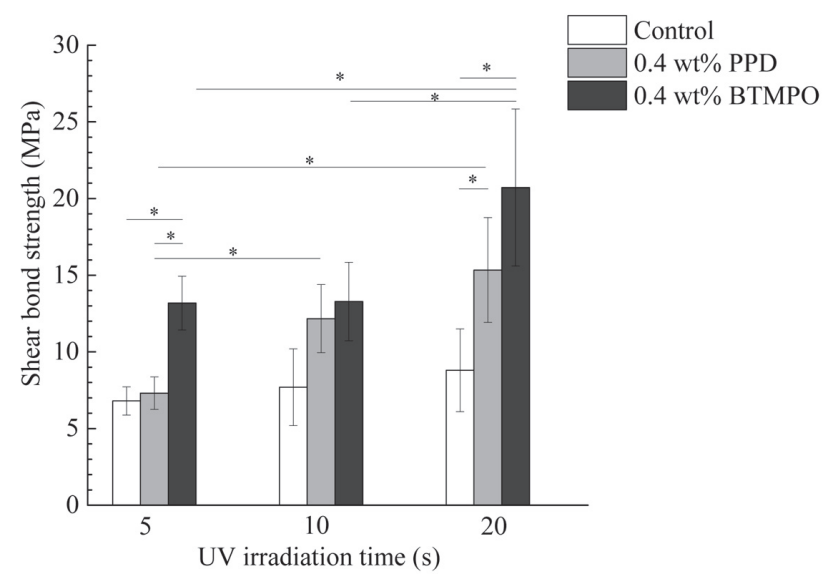

Fig. 5 Relationship between UV light irradiation time and compressive shear bond strengths.

Bars indicate standard deviation. Single asterisk indicates $p<0.05$ for specimen comparison. Control: pretreatment with only MMA (without UV initiators in MMA). 0.4 wt\% PPD and 0.4 wt\% BTMPO: MMA solutions containing 0.4\% UV initiators (i.e., PPD and BTMPO).

the bond strength of the control when irradiated for $20 \mathrm{~s}$. Moreover, the bond strength of the specimen precoated with BTMPO was significantly higher than that of the specimen pre-coated with PPD. The specimen precoated with $0.4 \mathrm{wt} \%$ BTMPO exhibited the highest bond strength for an irradiation time of $20 \mathrm{~s}$.

\section{DISCUSSION}

Several studies ${ }^{6-21)}$ have reported many surface treatments for PEEK, such as sandblasting, chemical treatment, and plasma and UV treatments that endow PEEK with adhesive properties. The bond strength of PEEK was significantly enhanced due to these surface treatments. Schmidlin et al. ${ }^{6}$ evaluated the shear bond strengths of sandblasted and acid-etched PEEKs as well as luting materials. The bond strengths of sandblasted and acid-etched PEEKs were 13.5 \pm 2.4 and 18.2 \pm 5.4 $\mathrm{MPa}$, respectively. Thus, the authors concluded that sandblasting and chemical treatment produced highly porous and permeable surfaces. Mechanical coupling could be achieved by their treatments. On the other hand, Huang et al. ${ }^{25)}$ reported that the aromatic ring between the two ether links in PEEK was sulfonated upon the treatment of PEEK with concentrated sulfuric acid, which probably resulted in chemical bonding.

Bötel et al. ${ }^{26)}$ observed that the bond strengths of PEEK were in the range of 19.6 to $33.8 \mathrm{MPa}$ after sandblasting and $\mathrm{Ar} / \mathrm{O}_{2}$ plasma treatments. Moreover, Shi et al. ${ }^{19)}$ reported that UV treatment effectively modified the chemical structure of a PEEK surface and improved its wettability. Thus, plasma and UV treatments improved the wettability of the PEEK surface and formed specific additional functional groups on the PEEK surface. The new functional groups that formed on the modified PEEK surface included COO and $\mathrm{OH}^{27,28)}$, which were expected to chemically bond with the adhesive resin.

We propose a novel surface treatment of PEEK in order to generate relatively high bond strength. Ikemura et $a l .^{23)}$ reported an excitation wavelength of approximately $400 \mathrm{~nm}$ for PPD and TMDPO, which is similar to the result obtained in this study. Meanwhile, a shorter wavelength of UV light has higher energy. According to a previous report ${ }^{28)}$, UV light (365 nm) can break the $\mathrm{C}-\mathrm{O}$ and $\mathrm{C}=\mathrm{O}$ bonds of PEEK, and the decomposed species can be expected to bond chemically to each other and to undergo polymerization upon exposure to UV light. For the above reasons, under UV light irradiation, the excitation wavelength of the photoinitiators was selected as approximately $365 \mathrm{~nm}$. The UV photoinitiators would produce radicals that facilitate the polymerization of MMA at a UV light excitation wavelength of $365 \mathrm{~nm}$. The FTIR results indicated the presence of PMMA, and thus suggested that a thin PMMA film was formed on the pre-coated PEEK surface.

Incidentally, the most common resins for using esthetic restorative materials in light-curing system are based on dimethacrylate or urethane dimethacrylate oligomers. These oligomers are viscous liquids ${ }^{29)}$. Therefore, it is difficult to dissolve UV initiators in these oligomers. However, MMA, a common monomer used to make complete dentures, is a liquid. UV initiators can easily dissolve in MMA. This is mainly because MMA is used as a solvent for UV initiators.

Based on the results, the mean bond strength increased from 7.3 MPa to high as 20.7 MPa, depending on the pre-coatings. Higher strengths were observed using lower concentrations of UV photoinitiators. According to the Beer-Lambert law for optical transmittance ${ }^{30)}$, a lower concentration results in less absorption, thereby increasing the penetration depth of UV light. Therefore, it was possible to decompose functional groups of the PEEK surface and perform light curing for both, the groups and MMA. Hence, relatively high bond strengths were obtained.

In general, the polymerization rate was proportional to the square root of the initiator concentration, and the polymerization degree was relatively high ${ }^{31}$. The mechanical properties of the polymer that was formed by UV light initiation were excellent at high concentrations of initiators. However, based on the principle of the Beer-Lambert law, a high concentration of initiators is associated with insufficient light penetration at the interface. Thus, any of the functional groups present at the surface of PEEK hardly decomposed. For these reasons, interfacial chemical bonding between the PEEK surface and PMMA polymerized by UV light irradiation is unfavorable. Therefore, the bond strengths associated with high-concentration initiators were not improved.

From the failure observation, wave-like traces of the resin formed by the tearing of the adhesive resin into polishing streaks were observed along the polishing 
streaks on the specimens pre-coated with PPD and TMDPO. Both, mechanical and chemical bonds, could occur on these specimens. In contrast, no polishing streaks were observed, and wave-like traces as well as small pieces of the resin were observed on the specimen pre-coated with BTMPO. The fracture pattern indicated the fracture of the resin layer, however, interface failure was not observed. Hence, strong chemical bonds could occur between the surface of PEEK and the resin. The pre-coating of the specimen with BTMPO led to the highest bond strength.

We also investigated the effect of irradiation time on the bond strength with the $0.4 \mathrm{wt} \%$ photoinitiator. The behaviors of the bond strengths of the specimens precoated with TMDPO and BTMPO were similar, which was attributed to the fact that both photoinitiators contained acylphosphine oxide and possessed similar molecular structures. Acylphosphine oxide has the distinct advantage of absorbing in the near UV-range to undergo a fast photolysis which generates reactive benzoyl and phosphinoly radicals ${ }^{32}$. Therefore, PPD and BTMPO were selected as the UV photoinitiators and were used to examine the effect of irradiation time on bond strength. The results postulate that longer irradiation times lead to higher bond strengths. Longer irradiation times supply additional light energy to the interface because photon irradiance is defined as the number of photons per unit area per unit time. Moreover, the radicals were rapidly generated. Thereby, they could facilitate an increased decomposition of $\mathrm{C}-\mathrm{O}$ or $\mathrm{C}=\mathrm{O}$ bonds in the PEEK polymer chains. Thus, graft polymerization would be expected to occur at the interface. In fact, Kyomoto et $a l .{ }^{33)}$ reported the graft polymerization of multifunctional poly(2-methacryloyloxyethyl phosphorylcholine) on the PEEK surface by utilizing a benzophenone (BP) photoinitiator and UV lamp with a wavelength of $350 \pm 50$ nm. Moreover, Deng et al. ${ }^{34)}$ indicated that UV-induced surface graft polymerization and chemical bonding with PMMA enhanced the bond strength. Thus, UV-assisted graft polymerization can be conducted using longer irradiation times, resulting in higher bond strengths.

This study does not estimate the effect of a specific thermal stress on the bond strength. Stawarczyk et al. ${ }^{13)}$ reported that the bond strengths of PEEK were slightly lower than those achieved after the thermal stress, however, their values were within clinical target ranges and were capable of achieving a sufficient level. Therefore, the bond strengths achieved after the thermal stress would be slightly low.

The pre-coating with MMA containing UV photoinitiators, as mentioned above, was simple in comparison with plasma and laser treatments, and can be introduced in clinical practice. This pre-coating method would contribute to increased bond strengths of PEEK.

\section{CONCLUSIONS}

After pre-coating the surfaces of PEEKs with MMA containing UV photoinitiators, they were irradiated with
UV light of wavelength $365 \mathrm{~nm}$. This pretreatment was performed before bonding the PEEKs to PEEK blocks using the MMA-based adhesive resin. The mean bond strength was $20.7 \pm 5.1 \mathrm{MPa}$ for pre-coating with MMA containing $0.4 \mathrm{wt} \%$ BTMPO, for $20 \mathrm{~s}$ of UV irradiation. In addition, cohesive failure of the adhesive resin was observed. Graft polymerization of PEEK could be induced on the PEEK surface. The use of this pre-coating led to relatively better bonding performance of PEEK.

\section{ACKNOWLEDGMENTS}

This research was supported by JSPS KAKENHI Grant Number 17K117776.

\section{REFERENCES}

1) Kurtz SM, Devine JN. PEEK biomaterials in trauma, orthopedic, and spinal implants. Biomaterials 2007; 28: 48454869.

2) Najeeb S, Zafar MS, Khurshid Z, Siddiqui F. Applications of polyetheretherketone (PEEK) in oral implantology and prosthodontics. J Prosthodont Res 2016; 60: 12-19.

3) Fujihara K, Huang ZM, Ramakrishna S, Satknanantham K, Hamada H. Feasibility of knitted carbon/PEEK composites for orthopedic bone plates. Biomaterials 2004; 25: 38773885 .

4) Stawarczyk B, Eichberger M, Uhrenbacher J, Wimmer T, Edelhoff D, Schmidlin PR. Three-unit reinforced Polyetheretherketone composite FDPs: Influence of fabrication method on load-bearing capacity and failure types. Dent Mater J 2015; 34: 7-12.

5) Liebermann A, Wimmer T, Schmidlin PR, Schere H, Loffler $\mathrm{P}$, Roos $\mathrm{M}$, et al. Physicomechanical characterization of Polyetheretherketone and current esthetic dental CAD/CAM polymers after aging in different storage media. J Prosthet Dent 2016; 115: 321-328.

6) Schmidlin PR, Stawarczyk B, Wieland M, Attin T, Hammerle CHF, Fischer J. Effect of different surface pre-treatments and luting materials on shear bond strength to PEEK. Dent Mater 2010; 26: 553-559.

7) Zhou L, Qian Y, Zhu Y, Liu H, Gan K, Guo J. The effect of different surface treatments on the bond strength of PEEK composite materials. Dent Mater 2014; 30: e209-e215.

8) Tsuka H, Morita K, Kato K, Kawano H, Abekura H, Tsuga K. Evaluation of shear bond strength between PEEK and resinbased luting material. J Oral Biosci 2017; 59: 231-236.

9) Hallmann L, Mehl A, Sereno N, Hammerle CHF. The improvement of adhesive properties of PEEK through different pre-treatments. Appl Surf Sci 2012; 258: 72137218.

10) Stawarczyk B, Beuer F, Wimmer T, Jahn D, Sener B, Roos $\mathrm{M}$, et al. Polyetheretherketone- A suitable material for fixed denture prostheses?. J Biomed Mater Res Part B 2013; 101: 1209-1216.

11) Kern M, Lehman F. Influence of surface conditioning on bonding to Polyetheretherketone (PEEK). Dent Mater 2012; 28: $1280-1283$.

12) Sproesser O, Schmidlin PR, Uhrenbacher J, Eichberger M, Roos M, Stawarczyk B. Work of adhesion between resin composite cements and PEEK as a function of etching duration with sulfuric acid and its correlation with bond strength values. Int J Adhes Adhes 2014; 54: 184-190.

13) Stawarczyk B, Jordan P, Schmidlin PR, Roos M, Eichberger $\mathrm{M}$, Gernet W, et al. PEEK surface treatment effects on tensile bond strength to veneering resins. J Prosthet Dent 2014; 112: 1278-1288. 
14) Zhang S, Awaja F, James N, Mckenzie DR, Ruys AJ. Autohesion of plasma treated semi-crystalline PEEK: Comparative study of argon, nitrogen and oxygen treatments. Colloids Surf A 2011; 374: 88-95.

15) Iqbal HMS, Bhowmik S, Benedictus R. Surface modification of high performance polymers by atmospheric pressure plasma and failure mechanism of adhesive bonded joints. Int J Adhes Adhes 2010; 30: 418-424.

16) Jha S, Bhowmik S, Bhatnagar N, Bhattacharya NK, Deka U, Iqbal HMS, et al. Experimental investigation into the effect of adhesion properties of PEEK modified by atmospheric pressure plasma and low pressure plasma. J Appl Polym Sci 2010; 118: 173-179.

17) Stawarezyk S, Bahr N, Beuer F, Wimmer T, Eichberger $\mathrm{M}$, Gernet $\mathrm{W}$, et al. Influence of plasma pretreatment on shear bond strength of self-adhesive resin cements to polyetheretherketone. Clin Oral Invest 2014; 18: 163-170.

18) Comyn J, Mascia L, Xiao G, Parker BM. Plasma-treatment of polyetherertherketone (PEEK) for adhesive bonding. Int J Adhes Adhes 1996; 16: 94-104.

19) Shi H, Sinke J, Benedictus R. Surface modification of PEEK by UV irradiation for direct co-curing with carbon fiber reinforced epoxy prepregs. Int J Adhes Adhes 2017; 73: 5157.

20) Mathieson I, Bradley RH. Improved adhesion to polymers by UV/ozone surface oxidation. Int J Adhes Adhes 1996; 16: 2931.

21) Niu YF, Yang Y, Li TY, Yao JW. Effects of UV irradiation and condensation on poly(ether-ether -ketone)/carbon fiber composites from nano-to macro-scale. High Perform Polym 2018; 30: 230-238.

22) Smith BGN, Wright PS, Brown D. The clinical handling of dental materials, 2th ed. Oxford: Butterworth-Heinemann Ltd; 1994. p. 208.

23) Ikemura K, Ichizawa K, Yoshida M, Ito S, Endo T. UV-VIS spectra and photoinitiation behaviors of acylphosphine oxide and bisacylphosphine oxide derivatives in unfilled, light- cured dental resins. Dent Mater J 2008; 27: 765-774.

24) Duan G, Zhang C, Li A, Yang X, Lu L, Wang X. Preparation and characterization of mesoporous zirconia made by using a poly (methyl methacrylate) template. Nanoscale Res Lett 2008; 3: 118-122.

25) Huang RYM, Shao P, Burns CM, Feng X. Sulfonation of poly(ether ether ketone)(PEEK): kinetic study and characterization. J Appl Polym Sci 2001; 82: 2651-2660.

26) Bötel F, Zimmermann T, Sütel M, Müller WD, Schwitalla AD. Influence of different low-pressure plasma process parameters on shear bond strength between veneering composites and PEEK materials. Dent Mater 2018; 34: e246-e254.

27) Ha SW, Hauert R, Ernst KH, Wintermantel E. Surface analysis of chemically-etched and plasma-treated polyetheretherketone (PEEK) for biomedical applications. Surf Coat Technol 1997; 96: 293-299.

28) Yousaf A, Farrukh A, Oluz Z, Tuncel E, Duran H, Dogan SY, et al. UV-light assisted single step route to functional PEEK surfaces. React Funct Polym 2014; 83: 70-75.

29) Powers JM, Wataha JC. Dental materials: Properties and manipulation. 10th ed. St. Louis: Elsevier; 2013. p. 164-165.

30) Valeur B. Molecular Fluorescence: Principles and Applications. 1st ed. Wiley-VCH Verlag GmbH, German; 2001. p 20-30.

31) Su WF. Principles of Polymer Design and Synthesis. SpringerLink, First Online; 09 October 2013. p 137-183.

32) Decker C, Zahouily K, Decker C, Nguyen T, Viet T. Performance analysis of acylphohphine oxides in photoinitiated polymerization. Polymer 2001; 42: 7551-7560.

33) Kyomoto M, Moro T, Yamane S, Hashimoto M, Takatori Y, Ishihara K. Poly(ether-ether-ketone) orthopedic bearing surface modified by self-initiated surface grafting of poly(2methacryloyloxyethyl phosphorylcholine). Biomaterials 2013; 34: 7829-7839.

34) Deng J, Wang L, Liu L, Yang W. Developments and new applications of UV-induced surface graft polymerizations. Prog Polym Sci 2009; 34: 156-193. 